\title{
New era for European Respiratory Society clinical practice guidelines: joining efficiency and high methodological standards
}

\author{
Marc Miravitlles (10) ${ }^{1}$, Thomy Tonia ${ }^{2}$, David Rigau ${ }^{3}$, Nicolas Roche $^{4}$, \\ Céline Genton ${ }^{5}$, Valérie Vaccaro ${ }^{5}$, Tobias Welte ${ }^{6}$, Mina Gaga ${ }^{7}$ and \\ Guy Brusselle 8,9
}

Affiliations: ${ }^{1}$ Pneumology Dept, Hospital Universitari Vall d'Hebron, CIBER de Enfermedades Respiratorias (CIBERES), Barcelona, Spain. 'Institute of Social and Preventive Medicine, University of Bern, Bern, Switzerland. ${ }^{3}$ Iberoamerican Cochrane Center, Barcelona, Spain. ${ }^{4}$ Service de Pneumologie et Soins Intensifs Respiratoires, Hôpital Cochin (AP-HP), Université Paris Descartes (EA2511), Paris, France. ${ }^{5}$ Scientific Activities Dept, European Respiratory Society, Lausanne, Switzerland. ${ }^{6}$ Respiratory Medicine, Medical School Hannover, Hannover, Germany. ${ }^{7}$ Respiratory Medicine Dept and Asthma Center, Athens Chest Hospital "Sotiria", Athens, Greece. ${ }^{8}$ Dept of Respiratory Medicine, Ghent University Hospital, Ghent, Belgium. ${ }^{9}$ Dept of Epidemiology and Dept of Respiratory Medicine, Erasmus Medical Center, Rotterdam, The Netherlands.

Correspondence: Marc Miravitlles, Pneumology Dept, Hospital Universitari Vall d'Hebron, Barcelona, Spain. E-mail: mmiravitllesđavhebron.net

Clinical practice guidelines have to respond to the needs of users http://ow.ly/Vui330iwPbY

Cite this article as: Miravitlles M, Tonia T, Rigau D, et al. New era for European Respiratory Society clinical practice guidelines: joining efficiency and high methodological standards. Eur Respir J 2018; 51: 1800221 [https://doi.org/10.1183/13993003.00221-2018].

One of the most important tasks of a scientific society is the development of clinical practice guidelines (CPGs). The objectives of CPGs are to improve the quality and efficiency of healthcare, to reduce unjustified variations in the practices of diagnosis and treatment, and to improve patient outcomes. In order to fulfil these objectives, CPGs have to be evidence-based and therefore need to be developed through an unbiased process, consisting of systematic reviews, a standardised evaluation of the evidence and transparent evidence to decision (EtD) process. The European Respiratory Society (ERS) is committed to the development of the highest quality CPGs [1] and adopted the GRADE (Grading of Recommendations Assessment, Development and Evaluation) approach [2], which ensures that the ERS CPGs meet most of the Institute of Medicine standards for defining "trustworthy" CPGs [3].

GRADE is a transparent, evidence-based approach to CPG development and it requires systematic reviews to be conducted for all the questions for which recommendations are going to be proposed [2, 4]. These questions should be formulated in the PICO format (patient, intervention, comparison, outcomes) and are identified by the panel of participants in the CPG. The panel itself needs to comprise all of the caregivers and stakeholders involved, including patients, experts, field professionals, policy-makers, etc. All of the panellists need to participate in the development of the questions to be considered. Since conducting

Received: Jan 312018 | Accepted: Feb 012018

Conflict of interest: T. Tonia reports acting as a European Respiratory Society (ERS) methodologist. D. Rigau reports acting as an ERS methodologist. N. Roche reports grants and personal fees from Boehringer Ingelheim, from Novartis and from Pfizer; personal fees from Teva, GSK, AstraZeneca, Chiesi, Mundipharma, Cipla, Sanofi, Sandoz, 3M, and Zambon, outside the submitted work. C. Genton is an employee of the ERS. V. Vaccaro is an employee of the ERS. 
thorough systematic reviews for each question requires time and resources, the number of PICO questions that can be addressed in a CPG is limited by necessity. Consequently, there is a risk of producing CPGs that are just answers to a list of a few questions, without having the connecting "lines between them". This type of guideline, albeit methodologically rigorous, may not be so useful for the practising clinician, who still has to connect the dots between the questions and make decisions on several aspects that may not be covered in the final document [5]. This situation may leave the clinician uninformed about relevant aspects of daily practice and will force the assumption of decisions for which there is not any hint on what might be the best option [6]. For the majority of these situations, the members of the panel are still in a better position to make a recommendation to the clinician, since they have accumulated a great deal of clinical experience and have dedicated time and resources to consider all the available information in the process of developing the CPG. However, the challenge is to make CPGs more useful (and user-friendly) to the practising clinician, while maintaining the rigour, the high scientific quality and minimising the risk of bias [7]. It has to be ensured that all recommendations are based on the best available evidence, even when thorough systematic reviews are not feasible for all questions. This evidence has to be complimented by clinical experience and the process of combining all the different information and reaching recommendations has to be fully transparent. Costs and applicability issues also need to be considered.

These concerns have been considered by other CPG developers. As an example, the American College of Chest Physicians (CHEST) has established what they call a hybrid process that includes evidence-based recommendations developed by the GRADE methodology [8] and, when the evidence is insufficient for a guideline, it can be combined with a Delphi process for consensus achievement resulting in trustworthy consensus statements [9]. CHEST recognises that clinicians seek guidance for some important problems for which insufficient research prevents the establishment of fully formalised evidence-based guidelines. For such cases, they have developed a structured approach including a systematic review of literature and the participation of rigorously vetted and entrusted experts [9]. Among other options to change CPGs development, SCHOENBERG et al. [10] proposed the new CORE (Convergence of Opinion on Recommendations and Evidence) process, by which they try to discriminate, early during guideline development, recommendations that should be formed by a systematic review and those that can be based upon expert consensus. The CORE process is a modified Delphi process. When it was applied to a panel of participants during CPG development it was observed that, when a threshold of $70 \%$ agreement was required for a given recommendation, more than $80 \%$ of the analysed questions yielded recommendations and the recommended courses of action were $98.2 \%$ concordant with those obtained previously for the same PICO question through the whole GRADE methodology [10]. Therefore, the authors of the study proposed the CORE process as an early step in guideline creation, and that questions which reach $70 \%$ agreement on a recommendation could be accepted, avoiding a lengthy systematic review [10]. However, this methodology may have some issues and would need further prospective validation [11].

Based on the discussions of the Guidelines Working Group and the Science Council of the ERS it was decided to implement a new process for the development of CPGs. The basis of the new process can be summarised as follows. 1) PICO questions and full systematic reviews will still be the focus of the guideline, and they will be used as the basis to provide recommendations following the GRADE methodology and according to the Institute of Medicine standards. 2) There are some relevant clinical questions that may not have been addressed in randomised trials or cannot be formulated as PICO questions. For instance, questions regarding the type and frequency of disease monitoring, specialist care referral, environmental, hygiene or lifestyle interventions, treatment selection in case of specific comorbidities or drug interactions, or when trying to establish a hierarchy of different drugs that might all be recommended for the same indication, without comparative head-to-head studies. In these situations, a summary of the best available evidence, other anticipated benefits or harms beyond study results, drug pharmacological properties or intervention characteristics, and factors such as acceptability, availability, feasibility and equity may well justify a recommendation without a full systematic appraisal of the evidence. 3) For both types of questions (i.e. PICO and non-PICO) the EtD framework will be used to clearly and transparently document what kind of information has been used in order to establish the recommendation [12]. 4) This process will result in more complete and useful documents that will include algorithms and decision trees, which can then be more efficiently translated into pocket-guideline format, apps, etc., facilitating optimal dissemination and implementation of the CPG. The aim is to help the clinician make the best decisions quickly and effectively, while keeping the high quality and evidence-based nature of the CPG. The new process for creation of CPGs is summarised in figure 1.

A practical example of how this new approach can be used to improve CPGs may arise from the recent ERS/American Thoracic Society CPG for prevention of exacerbations of chronic obstructive pulmonary disease (COPD), which was based on five PICO questions [13]. In this document, three different oral treatments were recommended for prevention of exacerbations of COPD with the same strength of 


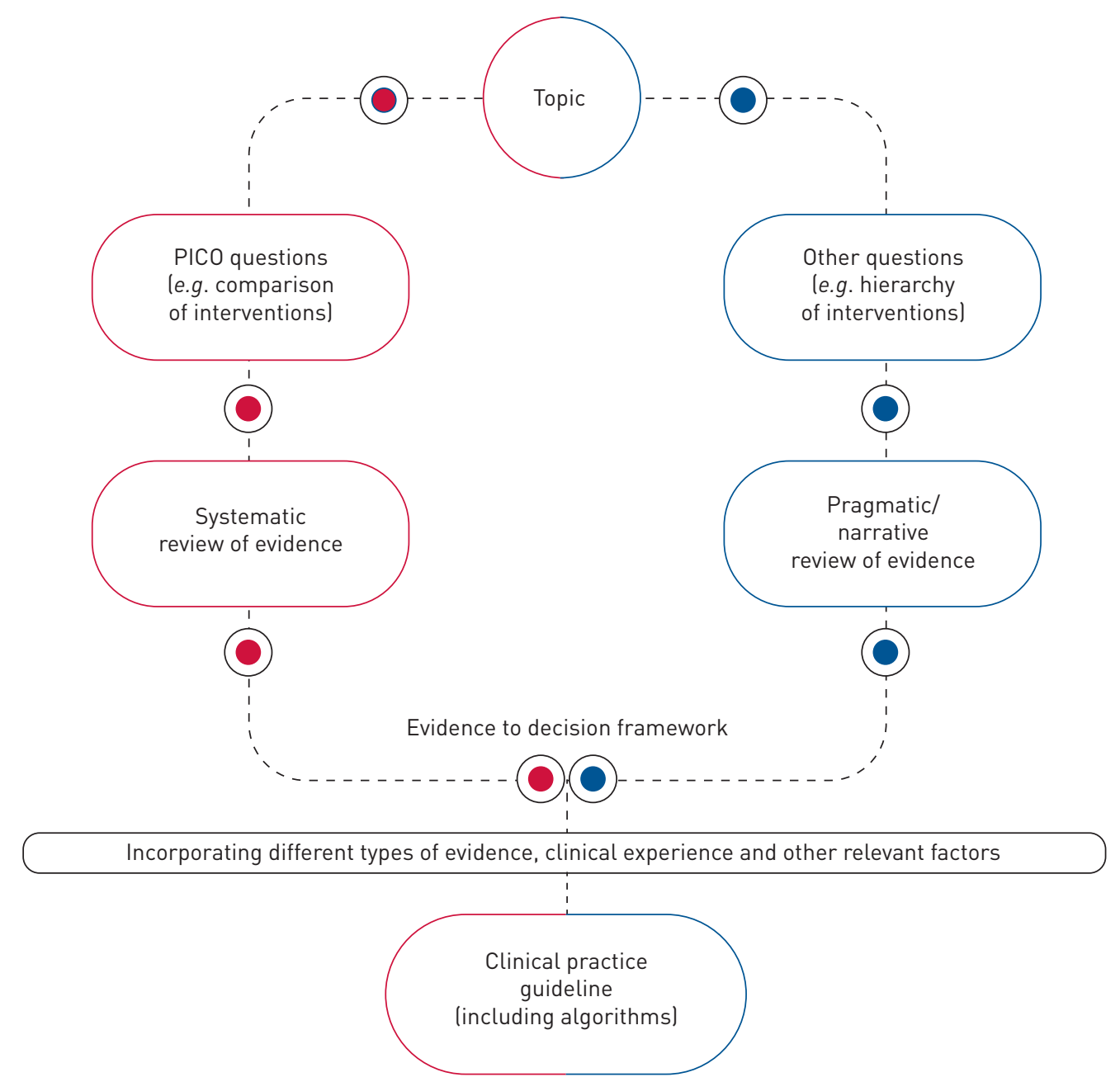

FIGURE 1 Overview of the new process for creation of clinical practice guidelines. PICO: patient, intervention, comparison, outcomes.

recommendation and level of evidence; therefore, which one should be used first? Or can they be used simultaneously? Or is there any preference according to different types (or phenotypes) of patients? Such relevant practical questions could not be addressed in a PICO format and could not easily and in a timely manner be evaluated through complete systematic reviews and by applying the full GRADE methodology. The answer to these practical non-PICO questions must be based on a literature review; however, this review will be narrative or pragmatic rather than systematic and it will be complemented by a description of usual clinical practice and the experience of the panel members. This new process of CPGs creation requires a priori selection of the questions to be developed based on a thorough systematic review and the application of the full GRADE approach and those that will be narrative, based on the type of question. The role of ERS methodologists will be crucial to ensure that the selection is unbiased and the EtD framework is followed and clearly documented [12].

The advantages of this new approach will be that the CPG will be more adapted to the complexity of clinical decision making, and should be better understood and accepted by clinicians; however, there is a risk if the right questions are not based on full systematic reviews and full GRADE evaluation. In particular, comparative questions must be addressed using the PICO format and via full systematic reviews of the literature and grading following GRADE, especially those from areas in which: 1) there might be a lot of new evidence; 2) there is controversy or disagreement between clinicians; or 3) when new, expensive, aggressive, burdensome or highly specialised interventions have to be evaluated. The questions selected to be answered in a more pragmatic or narrative way will be complimentary to the main PICO questions. They will either focus on background factors, such as aetiology, or on practical considerations, such as the hierarchy of administration of different drugs. By using the EtD framework for these questions [12], we will ensure that all the factors that are considered for each question will be clearly documented. Research 
evidence will always be used to answer our questions by conducting pragmatic literature reviews, and any other considerations taken into account will be transparently shown on the EtD. The implementation of this new approach will follow a careful monitoring and evaluation of the documents produced.

The balance between high-quality evidence-based CPGs and making these guidelines useful for clinicians can be challenging. At ERS, our ultimate target is improving patient care. Giving complete and useful guidance to clinicians in a timely manner is one step towards this goal. However, important challenges remain, so as to ensure that a CPG adequately answers the selected questions from the perspective of the end-users, and how to assess the impact of the CPG in the real-life care of the targeted patients.

\section{References}

1 Brusselle GG, Gaga M. ERS guidelines, statements and technical standards published in the ERJ in 2014: a year in review. Eur Respir J 2015; 45: 863-866.

2 Guyatt GH, Oxman AD, Kunz R, et al. Rating quality of evidence and strength of recommendations. Incorporating considerations of resources use into grading recommendations. BMJ 2008; 336: 1170-1173.

3 Committee on Standards for Developing Trustworthy Clinical Practice Guidelines. Clinical Practice Guidelines We Can Trust. Washington, Institute of Medicine, 2011. Date last accessed: December 10, 2017. www. nationalacademies.org/hmd/Reports/2011/Clinical-Practice-Guidelines-We-Can-Trust.aspx

4 Guyatt GH, Oxman AD, Kunz R, et al. GRADE guidelines: 8. Rating the quality of evidenced-indirectness. J Clin Epidemiol 2011; 64: 1303-1310.

5 Neumann I, Santesso N, Akl EA, et al. A guide for health professionals to interpret and use recommendations in guidelines developed with the GRADE approach. J Clin Epidemiol 2016; 72: 45-55.

6 Miravitlles M, Roche N, Cardoso J, et al. Chronic obstructive pulmonary disease guidelines in Europe: a look into the future. Respir Res 2018; 19: 11.

7 Joos G, Bush A, Burghuber OC, et al. Taken to task: what is and is not an appropriate response to an ERS guidelines task force? Eur Respir J 2017; 50: 1700952.

8 Diekemper RL, Patel S, Mette SA, et al. Making the GRADE: CHEST updates its methodology. Chest 2017; in press [https://doi.org/10.1016/j.chest.2016.04.018].

9 Lewis SZ, Diekemper R, Ornelas J, et al. Methodologies for the development of CHEST guidelines and expert panel reports. Chest 2014; 146: 182-192.

10 Schoenberg NC, Barker AF, Bernardo J, et al. A comparative analysis of pulmonary and critical care medicine guideline development methodologies. Am J Respir Crit Care Med 2017; 196: 621-627.

11 Krishnan JA. Developing clinical guidelines: 99\% faster is not enough. Am J Respir Crit Care Med 2017; 196: 543-544.

12 Alonso-Coello P, Oxman AD, Moberg J, et al. GRADE Evidence to Decision (EtD) frameworks: a systematic and transparent approach to making well informed healthcare choices. 2: Clinical practice guidelines. BMJ 2016; 353: i2089.

13 Wedzicha JA, Calverley PMA, Albert RK, et al. Prevention of COPD exacerbations: a European Respiratory Society/American Thoracic Society guideline. Eur Respir J 2017; 50: 1602265. 